\title{
Two micro-portal video-assisted thoracic surgery of right S8 segmentectomy with systemic lymphadenectomy
}

\author{
Honggang Liu\#, Liping Tong", Yuanyang Lai", Hongtao Duan", Xiaoping Dong, Yong Zhang, Jianyong \\ Sun, Xiaolong Yan \\ Department of Thoracic Surgery, Tangdu Hospital, The Air Force Medical University, Xi'an, China \\ \#These authors contributed equally to this work. \\ Correspondence to: Xiaolong Yan. Department of Thoracic Surgery, Tangdu Hospital, The Air Force Medical University, Xi'an, China. \\ Email: yanxiaolong@fmmu.edu.cn.
}

\begin{abstract}
Better management of lung cancer is crucial for improving the prognosis of such lethal malignancy, and surgical treatment is one of the most important strategies for early lung cancer. Anatomic segmentectomy, in particular, provides the complete resection of lesions with the retention of maximal normal lung tissue. Several different techniques for lung segmentectomy have been described previously, whereas how to perform with concerns of minimal invasiveness and better accessibility for surgeons familiar with single-direction video-assisted thoracic surgery (VATS) remains to be elucidated. In the presented case, we described the applications of our two-micro-portal video-assisted thoracic segmentectomy as well as the plug-in multi-edge cutting method to a patient with a mixed ground-glass nodule in the anterior basal segment of the right lower lobe, and systemic lymphadenectomy was performed following the pathological diagnosis of invasive adenocarcinoma. The patient was discharged after a 4-day well recovery and was without recurrence or metastasis after 6 months of follow-up. Considering that the two micro-portal VATS would be more accessible for surgeons who are already familiar with the single-direction VATS and could yield the similar minimal invasiveness and less pain as uniport VATS, we believe that the presented twomicro-portal video-assisted thoracic segmentectomy is technically safe and feasible in lung cancer treatment and is worthy of promotion.
\end{abstract}

Keywords: Two micro-portal; video-assisted thoracic segmentectomy; lung ground-glass nodules

Received: 15 April 2020; Accepted: 22 December 2020; Published: 25 February 2022.

doi: $10.21037 /$ ccts-20-89

View this article at: http://dx.doi.org/10.21037/ccts-20-89

\section{Introduction}

Lung cancer remains the most severe cancer in China (1). With the prevalence of low-dose computed tomography, more and more pulmonary nodules are found, especially the ground-glass nodules in the lungs (2). Among them, mixed ground-glass opacity (GGO) has a higher malignant tendency, more solid components indicating a higher possibility of invasive adenocarcinoma. The surgical management for GGO are mainly sub-lobar resections consisting of wedge resection and anatomical segmentectomy. Wedge resection is mainly used for pure GGO in the outer third of the lung, whereas anatomical segmentectomy not only provides much more accurate but has advantages on inter-lobular and inter-segmental lymphadenectomies. For the concerns of minimal invasiveness and better accessibility for surgeons familiar with single-direction video-assisted thoracic surgery (VATS), this article introduces our surgical experience of two micro-portal video-assisted thoracic segmentectomy and lymphadenectomy (3), and the innovative application of the plug-in multi-edge cutting method for inter-segmental plane dealing.

\section{Clinical summary}

The 58-year-old man presented a 13 -mm mixed ground- 

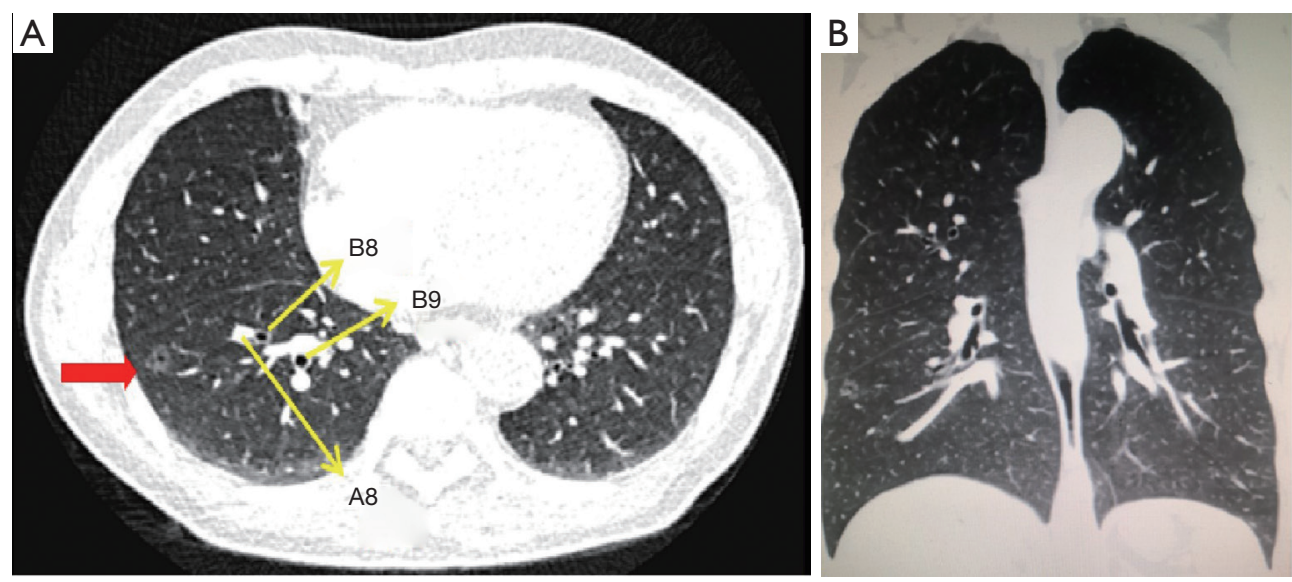

Figure 1 A 13-mm mixed ground-glass nodule (red arrows) with empty bubbles was presented at right S8. Axal (A) and coronal (B) views.
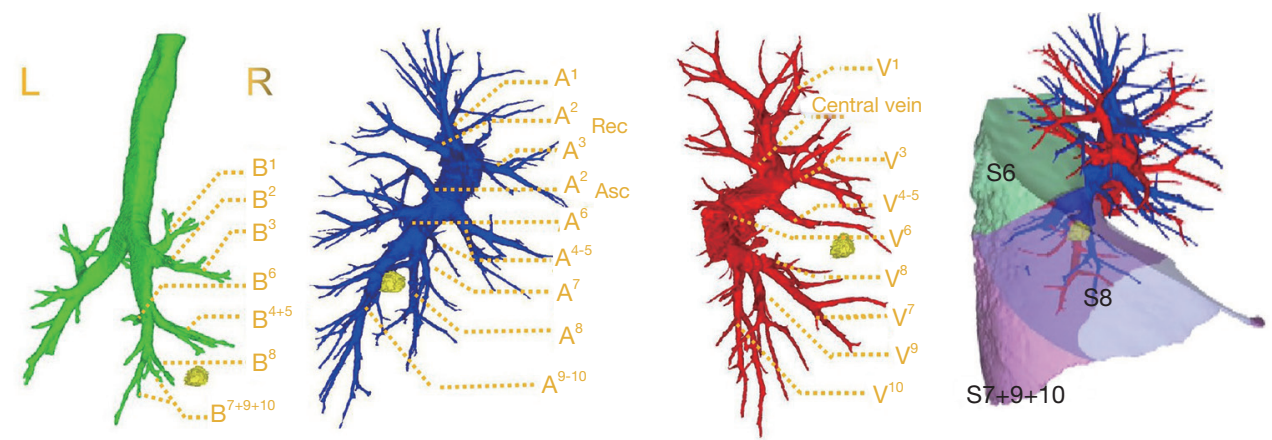

Figure 2 3D-CTBA reconstruction showed no variations of the trachea, artery and vein and the lesion was located at S8 of the right lower lobe.

glass nodule with empty bubbles in the anterior basal segment of the right lower lobe (Figure 1). After the 2-month follow-up of quiescence, the patient was admitted for further treatment. The patient was absent from hypertension, diabetes, tuberculosis, and autoimmune diseases, etc.; no abnormality was found in bronchoscopy, lung function, blood gas analysis, colour Doppler echocardiography, electrocardiogram, and blood tests. There was no sign of metastatic disease preoperatively. All procedures performed in this study were in accordance with the ethical standards of the institutional and/or national research committee(s), and with the Helsinki Declaration (as revised in 2013). Written informed consent was obtained from the patient for publication of this study and accompanying images. A copy of the written consent is available for review by the editorial office of this journal.

\section{Surgical techniques}

Three-dimensional computed tomography bronchography and angiography (3D-CTBA) via a Materialise Mimics 17.0 software was utilized to define the location of the lesion and the anatomy of the relevant bronchial and vascular branches, to conclude bronchus segmentalis and blood vessels that need to be cut, and to formulate the surgical plan (Figure 2). We determined the lesion was located in the anterior basal segment of the right lower lobe and planned to perform two micro-portal video-assisted thoracic segmentectomy at S8 of the right lower lobe with systemic lymphadenectomy $(4,5)$. The surgical procedure is shown below (Video 1).

\section{Anaesthesia, positioning, and port placement}

Compound fentanyl and double-lumen endobronchial tube 


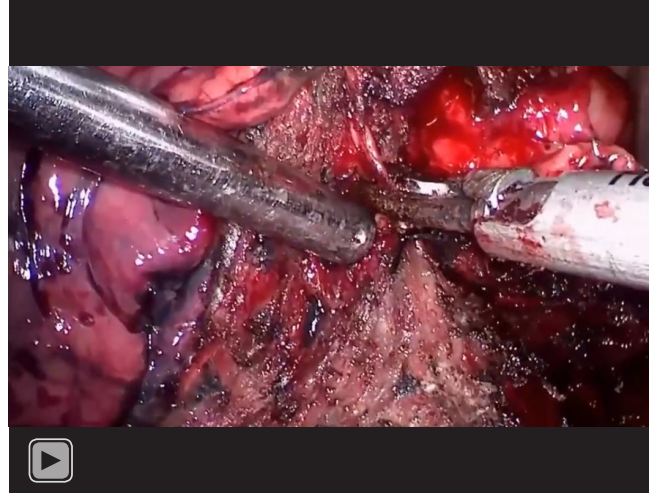

Video 1 Two-micro-portal video-assisted thoracic segmentectomy at right $\mathrm{S} 8+\mathrm{LN}$ dissection. $\mathrm{LN}$, lymph node.

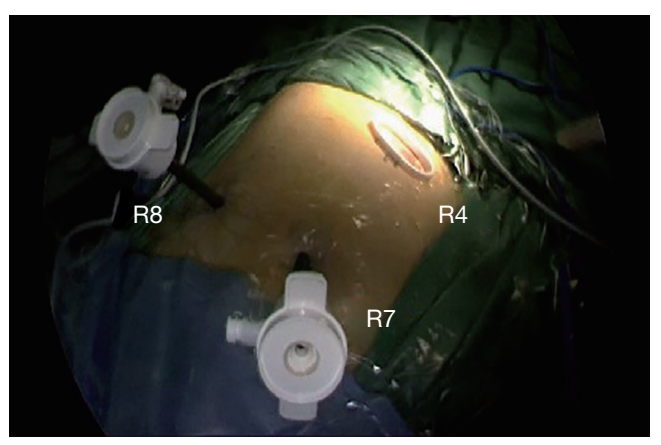

Figure 3 Incisions during operation.

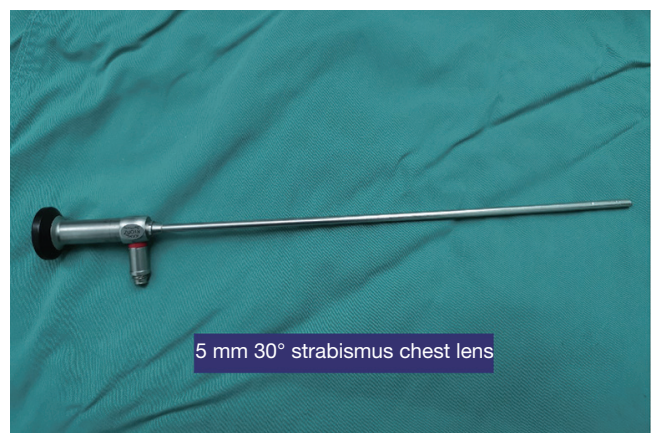

Figure 4 - $\mathrm{mm} 30^{\circ}$ strabismus chest lens.

were applied for anaesthesia and ventilation, respectively. The patient was placed in the left lateral decubitus position with arms stretched to $90^{\circ}$, and the table was $30^{\circ}$ flexed to enlarge the intercostal space for the protection of the intercostal neurovascular bundles. The main operation incision of $2-\mathrm{cm}$ length was performed in the fourth intercostal space at the anterior axillary line; while $0.5-\mathrm{cm}$ secondary operating port located in the eighth intercostal space at the posterior axillary line and the viewing port $(0.5 \mathrm{~cm})$ the seventh intercostal space at the midaxillary line (Figure 3).

By the $7^{\text {th }}$ intercostal endoscopic exploration $(5 \mathrm{~mm}$ $30^{\circ}$ strabismus chest lens, Figure 4), the lesion was marked at $\mathrm{S}^{8}$ of the right lower lobe according to thin-layer CT anatomical labelling (Figure $5 A$ ) (6). The inferior pulmonary ligament was exposed and loosen following the removal of $9^{\text {th }}$ group lymph nodes (Figure 5B); the interlobular fissure was cut and the 11th group lymph nodes were resected afterwards (Figure 5C). $\mathrm{A}^{6}, \mathrm{~A}^{7}, \mathrm{~A}^{8}$, and $\mathrm{A}^{9-10}$ being dissected and the $12^{\text {th }}$ group lymph nodes being ablated (Figure $5 D$ ), the $\mathrm{A}^{8}$ was confirmed (Figure $5 E$ ) and transected via a $3-\mathrm{cm}$ EndoGIA linear white stapler (Covidien, Mansfield, MA, USA). The $B^{8}$ was cut and sutured with a $6.0 \mathrm{~cm}$ purple stapler (Covidien, Mansfield, MA, USA) after exposure (Figure $5 F$ ). The lung was reventilated with pure oxygen, and 5 minutes later, a clear inflation-deflation boundary was formed between the still inflated $S^{8}$ and the collapsed $\mathrm{S}^{6}, \mathrm{~S}^{7}$ and $\mathrm{S}^{9-10}$ (Figure $5 G$ ) and was then marked by electrocautery. The segmental porta was exposed along with the segmental plane and the inter-segment vein (Figure 5H), and $V^{8}$ a was ligatured with the sparing of $V^{8} b$ and $V^{7} b$ (Figure $5 I$ ). $\mathrm{S}^{8}$ was then removed via $6.0-\mathrm{cm}$ green staplers (Covidien, Mansfield, (MA, USA) according to the plugin multi-side cutting method (Figure 57), and the lesion was confirmed (Figure $5 K$ ). The segmentectomy concluded after confirmation of no active bleeding and accuracy of $\mathrm{A}^{8}$, $\mathrm{B}^{8}, \mathrm{~V}^{8}$ a stumps; the adjacent lung tissue is well recruited without shrinkage (Figure $5 L$ ).

\section{Radical mediastinal lymphadenectomy}

Systemic lymphadenectomy was performed after pathological confirmation of invasive adenocarcinoma. First, the posterior mediastinal pleura was dissected along the oesophagus, right main bronchus and hilar, and the $7^{\text {th }}$ group lymph nodes were removed with the protection of the vagus nerve (Figure 6). Second, the mediastinal pleura was opened along with the azygos vein, and the $2^{\text {nd }}$ and $4^{\text {th }}$ groups of lymph nodes were excised (Figure 7). No air leakage having been confirmed, the margins were applied with absorbable regenerated oxidized fibre membrane to reduce risks of further bleeding and air leakage. 24F chest drainage tubes were placed at the posterior mediastinum from the $8^{\text {th }}$ intercostal space and to the top of the chest cavity at the $7^{\text {th }}$ intercostal space (Figure 8). 

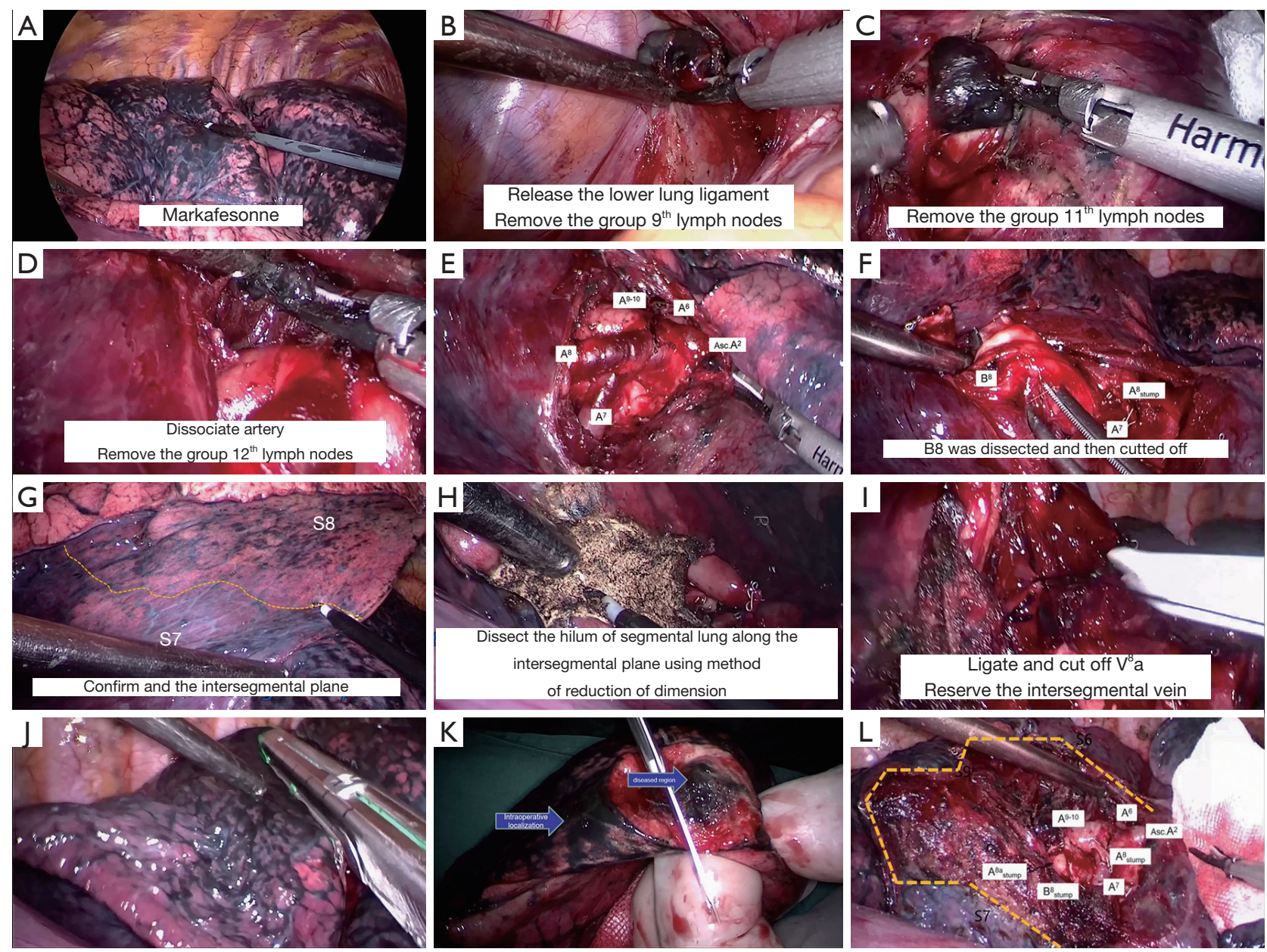

Figure 5 Procedures of $\mathrm{S}^{8}$ segmentectomy. (A) Mark of lesion; (B) $9^{\text {th }}$ group lymph nodes removal; (C) $11^{\text {th }}$ group lymph nodes removal; (D) $12^{\text {th }}$ group lymph nodes removal; (E) dissection of $\mathrm{A}^{6}, \mathrm{~A}^{7}, \mathrm{~A}^{8}, \mathrm{~A}^{9-10}$; (F) dissection of $\mathrm{B}^{8} ;(\mathrm{G})$ intersegmental plane confirmation; (H) dissection of segmental porta; (I) dissection and ligature of $\mathrm{V}^{8} \mathrm{a}$; (J) resection of $\mathrm{S}^{8}$ using the plug-in multi-side cutting method; (K) confirmation of lesion; (L) anatomical landmarks after S8 excision.

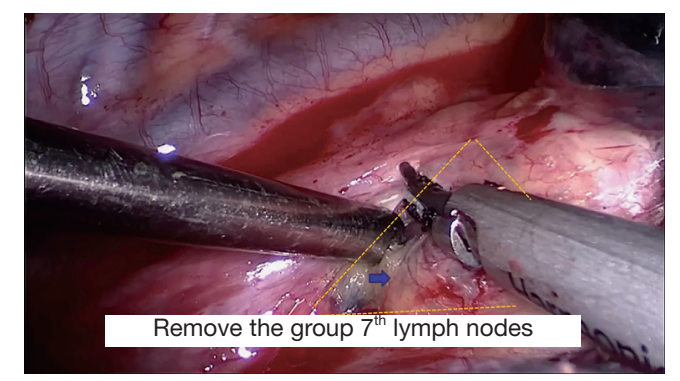

Figure 6 Removal of the $7^{\text {th }}$ group lymph nodes.

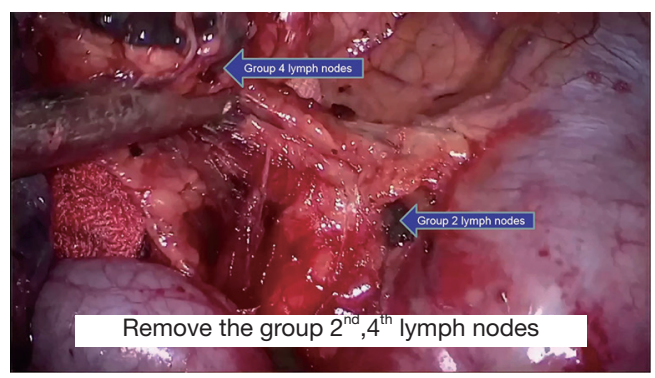

Figure 7 Removal of the $2^{\text {nd }}$ and $4^{\text {th }}$ groups of lymph nodes. 


\section{Postoperative management}

The operation lasted 120 minutes, the total number of lymph nodes dissected was 13 , and the intraoperative blood loss was $150 \mathrm{~mL}$. Postoperative analgesia pump, infection prevention, and phlegm treatment were routinely given; the patient was observed in the intensive care unit for 1 day, the postoperative

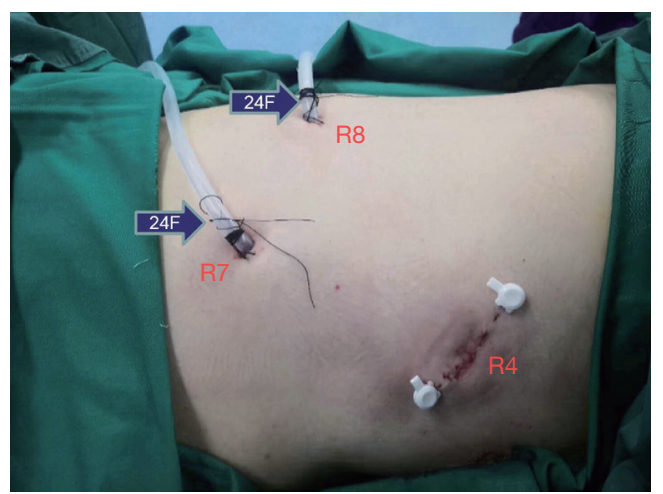

Figure 8 Incisions and chest tubes after surgery. The incision of the fourth intercostal space was about $2 \mathrm{~cm}$. drainage volume was $150 \mathrm{~mL}$ on the first day, $120 \mathrm{~mL}$ on the second day, and $30 \mathrm{~mL}$ on the third day. No obvious air leakage from the chest tube was found when coughing. The pain scores on days $1-3$ were 4,4 , and 3 respectively; chest $\mathrm{X}$-rays showed a good recruitment manoeuvre of the residual right lung, and the drainage tubes were removed on the third day after surgery. The postoperative hospital stay was 4 days, and no complications occurred. The postoperative pathological diagnose was invasive adenocarcinoma in stage pT1aN0M0 of the anterior basal segment of the right lower lobe, mainly LPA (Figure 9).

\section{Comments}

Due to the minimal trauma and technical maturity, VATS is recognized by most experts at home and abroad as one of the standard surgical procedures for early lung cancer $(7,8)$. At present, the most preferential mature incision options for VATS are triple-portal and uniportal mode. The former is beyond the limitation of the operating angle, making it more convenient for resections and more efficient (1). The uniportal VATS only makes one cut in a single
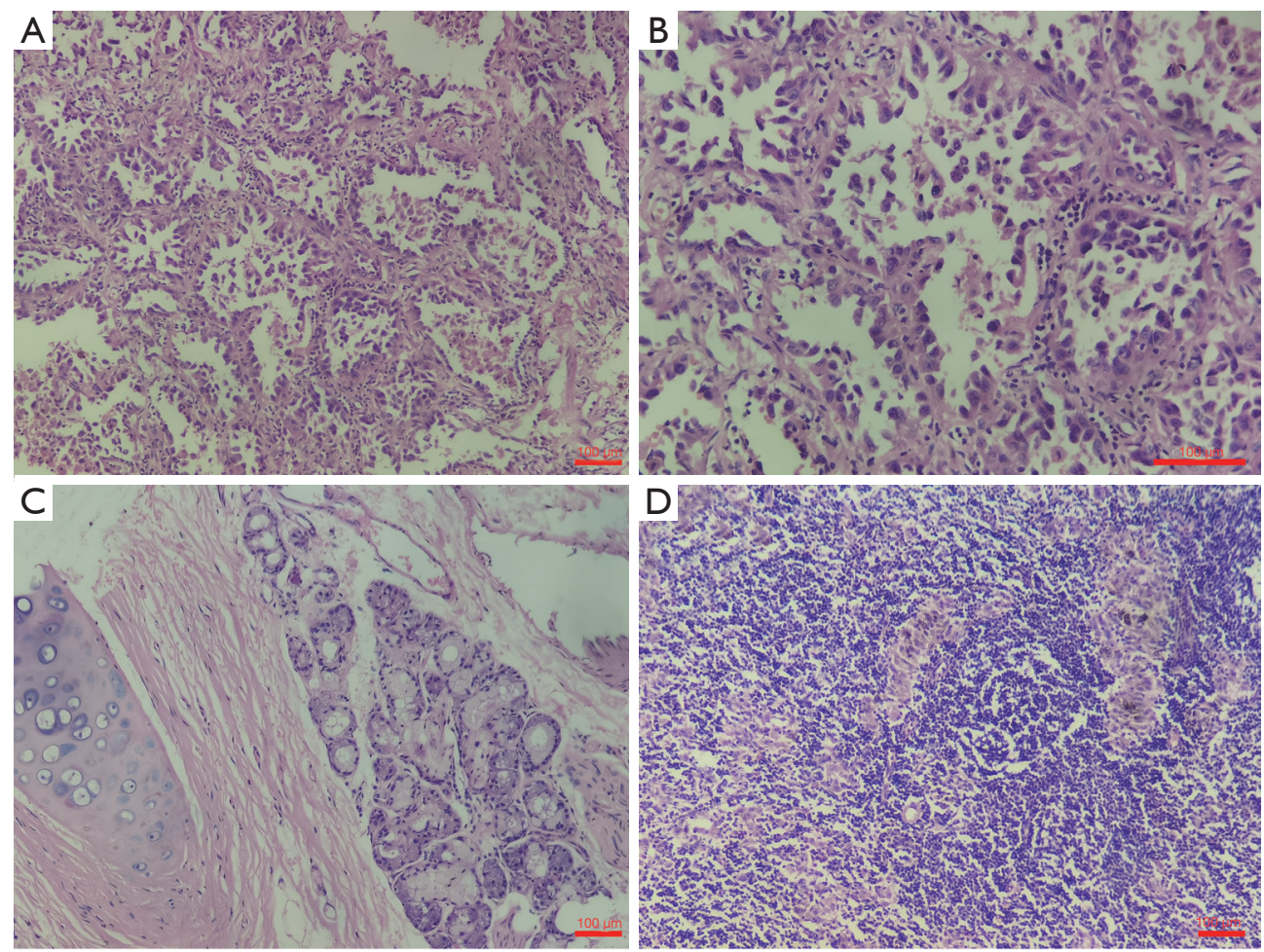

Figure 9 Invasive adenocarcinoma of anterior basal segment of the right lower lobe, mainly LPA, no pleural invasion. Bronchial stump and lymph nodes (No. 2, 4, 7, 9, 10,11, 12) were negative. HE staining. LPA, left pulmonary artery. 
intercostal space, so the postoperative incision looks better and the pain is less severe (9); however, for the internal operating angle being limited and higher requirements of instruments, this surgical technique is much more complicated and is not very suitable for beginners $(10,11)$. Our two micro-portal single-direction VATS model may have some unique features as it combines the advantages of the two and avoids their shortcomings. To begin with, the two micro-portal VATS would be more accessible for surgeons who are already familiar with the singledirection VATS, making the learning curve be smoother than that of uniport thoracoscopy. Next, unlike the uniport thoracoscopy, specific instruments are not necessarily required for better operating direction. Moreover, the similar minimal invasiveness and less pain as uniport VATS could be achieved by the two micro-portal VATS; in the present case, overall operation time was 120 minutes and the sum of incisions length was around $4 \mathrm{~cm}$.

We introduced the plug-in multi-side cutting method to deal with the intersegmental plane after recognition via differential inflation. The segmental bronchus and vessels having been well defined, staples were inserted under the intersegmental plane and multiple edges were made along the intersegmental vein and plane until the target segment was resected. In this case, the plugin multi-side cutting method (Figure $6 L$ ) could reduce the occurrence of bronchial injury, thermal injury and segmental portal vein injury, avoid bursting of staples, thus lessening persistent postoperative air leakage, chest haemorrhage, hemoptysis, intractable cough and other symptoms and shortening the hospital stay and accelerating the rehabilitation.

The two micro-portal VATS could optimize the perioperative process by the shorter length of the surgical incision, the single-direction resection "from back down to front up" and the plug-in multi-side cutting method for intersegmental plane management. It contributes to less pain, earlier extubation, and shorter hospital stay; it is in line with the concept of enhanced recovery after surgery (ERAS) (12). In summary, the two-micro-portal VATS is safe, effective, and worth promoting.

\section{Acknowledgments}

We gratefully acknowledge the invaluable cooperation of all the members of the Department of Thoracic Surgery, Tangdu Hospital, The Air Force Medical University, Xi'an, Shaanxi Province, China. The video was awarded second prize in the First International Lung Surgery Competition (2019 Masters of Lung Surgery).

Funding: This work was supported by grant from the Medical Technical Special Foundation of Tangdu Hospital (Grant No. TC201902).

\section{Footnote}

Peer Review File: Available at https://ccts.amegroups.com/ article/view/10.21037/ccts-20-89/prf

Conflicts of Interest: All authors have completed the ICMJE uniform disclosure form (available at https://ccts.amegroups. com/article/view/10.21037/ccts-20-89/coif). The authors have no conflicts of interest to declare.

Ethical Statement: The authors are accountable for all aspects of the work in ensuring that questions related to the accuracy or integrity of any part of the work are appropriately investigated and resolved. All procedures performed in this study were in accordance with the ethical standards of the institutional and/or national research committee(s), and with the Helsinki Declaration (as revised in 2013). Written informed consent was obtained from the patient for publication of this study and accompanying images. A copy of the written consent is available for review by the editorial office of this journal.

Open Access Statement: This is an Open Access article distributed in accordance with the Creative Commons Attribution-NonCommercial-NoDerivs 4.0 International License (CC BY-NC-ND 4.0), which permits the noncommercial replication and distribution of the article with the strict proviso that no changes or edits are made and the original work is properly cited (including links to both the formal publication through the relevant DOI and the license). See: https://creativecommons.org/licenses/by-nc-nd/4.0/.

\section{References}

1. Chen $W$, Zheng R, Baade PD, et al. Cancer statistics in China, 2015. CA Cancer J Clin 2016;66:115-32.

2. Jiang G, Chen C, Zhu Y, et al. Shanghai Pulmonary Hospital Experts Consensus on the Management of Ground-Glass Nodules Suspected as Lung Adenocarcinoma (Version 1). Chinese Journal of Lung Cancer 2018;21:147-59.

3. Tong L, Zheng X, Liu H, et al. Clinical efficacy of two micro-portal video-assisted thoracic surgery in pulmonary 
lobectomy for lung cancer. Chinese Journal of Clinical Thoracic and Cardiovascular Surgery 2019;26:369-73.

4. Weibing W, Lijun T, Quan Z, et al. The application of three-dimensional computed tomography angiography during thoracoscopic complex pulmonary segmentectomy. Chinese Journal of Thoracic and Cardiovascular Surgery 2015;31:649-52.

5. Chao S, Shichun L, Xiaolin W, et al. Application of Threedimensional Computed Tomography Bronchography and Angiography in Thoracoscopic Anatomical Segmentectomy. Chinese Journal of Minimally Invasive Surgery 2019;19:115-7.

6. Surgery Tegoltospsnptv-at. Consensus for localization technique of subcentimerter pulmonary small nodule prior to video-assisted thoracoscopic surgery (version 2019). Chinese Journal of Clinical Thoracic and Cardiovascular Surgery 2019;26:109-13.

7. Detterbeck F, Molins L. Video-assisted thoracic surgery and open chest surgery in lung cancer treatment: present and future. J Vis Surg 2016;2:173.

doi: $10.21037 /$ ccts-20-89

Cite this article as: Liu H, Tong L, Lai Y, Duan H, Dong X, Zhang Y, Sun J, Yan X. Two micro-portal video-assisted thoracic surgery of right S8 segmentectomy with systemic lymphadenectomy. Curr Chall Thorac Surg 2022;4:11.
8. Guerrero WG, González-Rivas D. Multiportal videoassisted thoracic surgery, uniportal video-assisted thoracic surgery and minimally invasive open chest surgeryselection criteria. J Vis Surg 2017;3:56.

9. Harris CG, James RS, Tian DH, et al. Systematic review and meta-analysis of uniportal versus multiportal videoassisted thoracoscopic lobectomy for lung cancer. Ann Cardiothorac Surg 2016;5:76.

10. Xie D, Wang H, Fei K, et al. Single-port video-assisted thoracic surgery in 1063 cases: a single-institution experience†. Eur J Cardiothorac Surg 2016;49:131-6.

11. Wang L, Liu D, Lu J, et al. The feasibility and advantage of uniportal video-assisted thoracoscopic surgery (VATS) in pulmonary lobectomy. BMC Cancer 2017;17:75.

12. Guo-wei C, Wei-min L, Lun-xu L. Enhanced Lung Rehabilitation after Surgery: Peri-operative Processes Optimization Is Necessary. Chinese Journal of Clinical Thoracic and Cardiovascular Surgery 2016;23:216-20. 